\title{
The synthesis of potential DNA intercalaters. 1. Heterocycles from the reaction of aryl bis-isothiocyanates
}

\author{
Ali Reza Molla Ebrahimlo ${ }^{\mathrm{a}, \mathrm{b}}$ and Jabbar Khalafy ${ }^{\mathrm{a}, *}$ \\ ${ }^{a}$ Chemistry Department, Urmia University, Urmia 57159, Iran \\ ${ }^{b}$ Chemistry Department, Islamic Azad University, Khoy Branch, Khoy, Iran \\ E-mail: j.khalafi@mail.urmia.ac.ir
}

\begin{abstract}
1,3-Bis(isoxazol-3-ylamino)benzenes, substituted on isoxazole-nitrogen with a pyrimidine group, react with triethylamine in ethanol under reflux to afford the corresponding 2indolylaminoimidazopyrimidine derivatives.
\end{abstract}

Keyword: Aryl bis-isothiocyanates, 1,3-bis(isoxazol-3-ylamino)benzenes, 2-chloropyrimidine, base induced rearrangement, indolylaminoimidazopyrimidine

\section{Introduction}

Prager and co-workers have reported ${ }^{1}$ that 2-aryl-3-arylaminoisoxazol-5(2H)-ones undergo solvolysis in the presence of potassium carbonate to form either imidazopyridines or indoles, and have suggested the reaction proceeds via the formation of 1,3-dipoles that undergo intramolecular cyclisation.

We have also reported ${ }^{2}$ that arylaminoisoxazol-5(2H)-ones, substituted on nitrogen with a nitropyridine group (1), react with triethylamine in ethanol under reflux condition to provide a convenient synthesis of ethyl 2-arylaminoimidazo[1,2-a]pyridine-3-carboxylates (2) (Scheme 1).

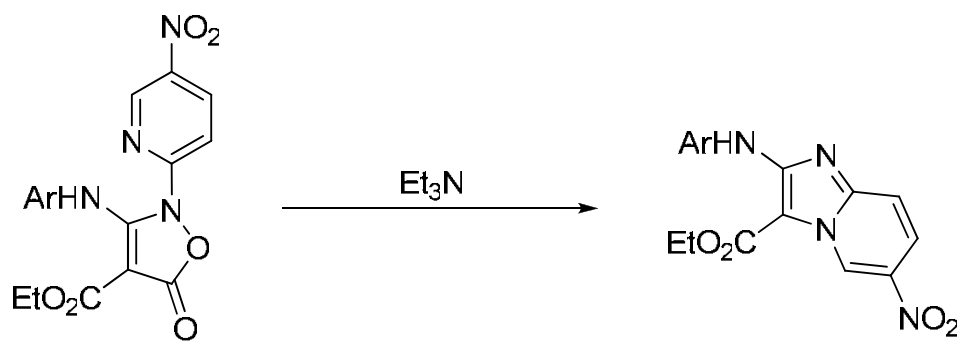

(1)

(2)

\section{Scheme 1}


We have recently extended this work by noting ${ }^{3,4}$ that the reaction of 3-(4-substitutedphenyl)aminoisoxazol-5(2H)-ones (3) and (4) with triethylamine leads to the formation of indoles (5), or imidazobenzothiazoles (6) respectively, and carbon dioxide, an outcome that is formally the same as that achieved by photolysis or pyrolsis ${ }^{5}$ (Scheme 2).<smiles>[X]c1ccc(Nc2c(C(=O)OCC)c(=O)on2-c2nc3ccccc3o2)cc1</smiles>

(3)<smiles>[X]c1ccc(Nc2c(C(=O)OCC)c(=O)on2-c2nc3ccccc3s2)cc1</smiles>

(4)

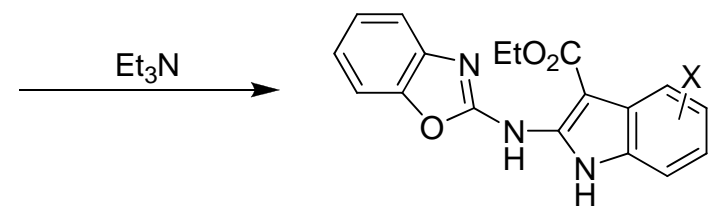

(5)

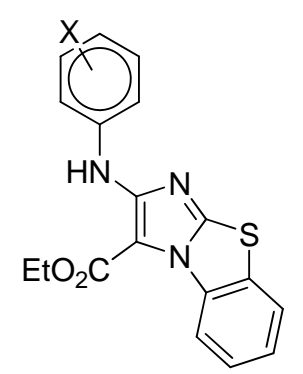

(6)

\section{Scheme 2}

In this paper we report the synthesis of new 1,3-bis(isoxazol-3-ylamino)benzenes and their isoxazolyl-N-substituted derivatives, and the rearrangement of the latter in the presence of triethylamine in ethanol under reflux to produce the indolylaminoimidazopyrimidine derivatives (Scheme 3). The reaction is formally a hybrid of the two reaction pathways noted previously, and provides suitable synthetic intermediates for a series of new heterocycles that could be expected to have pharmaceutical applications. ${ }^{6,7}$ 
<smiles>[R]c1c(NC(=S)C(C(=O)OCC)C(=O)OCC)cccc1NC(=S)C(C(=O)OCC)C(=O)OCC</smiles>

$7 a ; b$

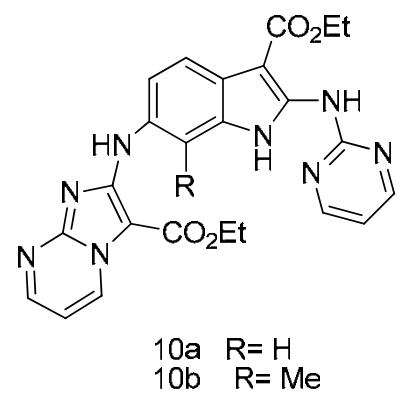

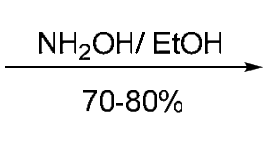

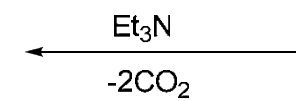

$\mathrm{R}=\mathrm{H}, \mathrm{Me}$<smiles>CCOC(=O)C1=C(Nc2cccc(Nc3[nH]oc(=O)c3C(=O)OCC)c2P)ONO1</smiles>

$8 a, b$

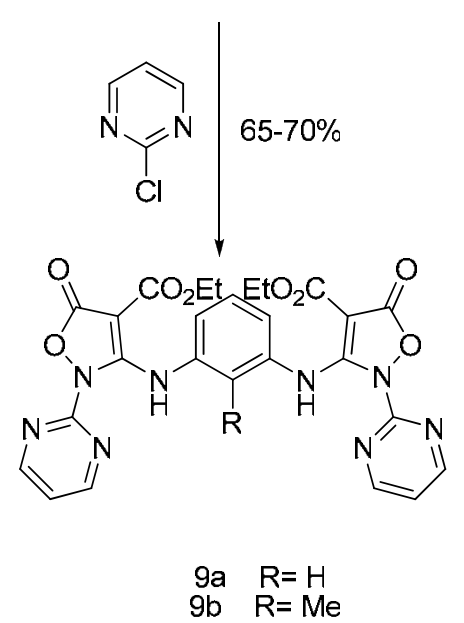

\section{Scheme 3}

\section{Results and Discussion}

The isoxazolones 8a,b were prepared by reaction of the corresponding thiocarbamates $\mathbf{7 a , b}$ with hydroxylamine by the general method of Worrall ${ }^{8}$ (Scheme 3 ).

$\mathrm{N}$-arylation of 8a,b with two equivalent of 2-chloroprymidine gave the desired N-substituted compounds 9a,b (Scheme 3). While the formation of 9a,b appear trivial, the reaction generally proceeded best in the absence of solvent, by heating the required reagent under nitrogen at $130^{\circ}$ $\mathrm{C}$ for $30 \mathrm{~min}$.

The rearrangement of bis pyrimidylisoxazolones 9a,b proceeded in refluxing ethanol for 3$12 \mathrm{~h}$ in the presence of triethylamine or potassium carbonate(Scheme 3 ).

The mechanism of the rearrangement is consistent with the previous suggestions ${ }^{1,9}$ for the formation of indoles and imidazopyrimidines from isoxazolones under basic conditions. We were unable to find any evidence for the formation of the bis indole (12), a plausible alternative product from the presumed penultimate intermediate (11) (Scheme 4). Presumably, subtle electron distribution within (11) dictates the direction of the final ring forming reaction. 

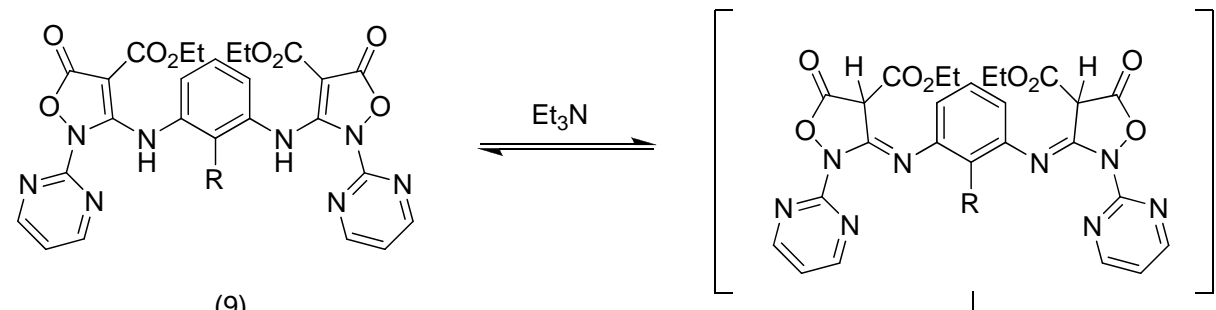

(9)
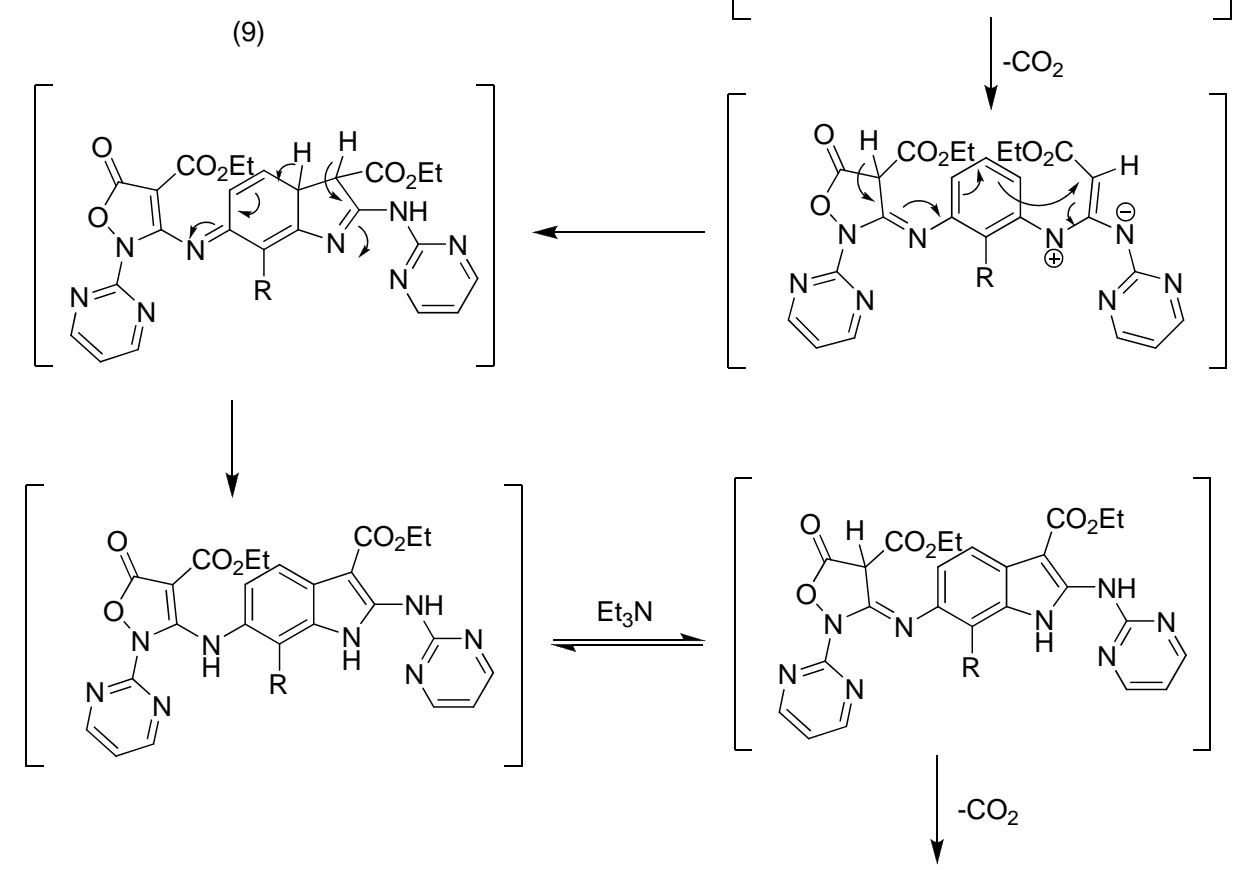

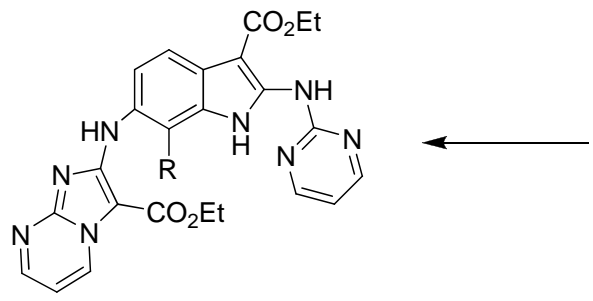

(10)

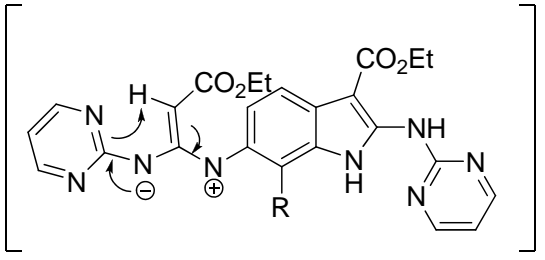

(11)<smiles>[R]c1c2[nH]c(Nc3ncccn3)c(C(=O)OCC)c2cc2c(C(=O)OCC)c(Nc3ncccn3)[nH]c12</smiles>

(12)

$\mathrm{R}=\mathrm{H}, \mathrm{Me}$

Scheme 4

\section{Conclusion}

The base catalysed rearrangements of 3-arylaminoisoxazolones substituted on N-2 with a diazine group appears to be generally applicable to the synthesis of indolaminoimidazopyrimidines, a 
class of molecule that has not been previously prepared and because of their multiple $\mathrm{H}$ bonding and acceptor sites, could be expected to intercalate with DNA. ${ }^{6,7}$. They would also serve as intermediates for new planar polycyclic heterocycles.

\section{Experimental Section}

General procedures. Freshly distilled solvents were used throughout, and anhydrous solvents were dried according to Perrin and Armarego. ${ }^{10}$ Melting points were determined on a Philip Harris C4954718 apparatus and are uncorrected. Infrared spectra were recorded on a Thermonicolet (Nexus 670) FT-infrared spectrometer, using sodium chloride cells and measured as film or $\mathrm{KBr}$ disks. ${ }^{1} \mathrm{H}(300 \mathrm{MHz})$ and ${ }^{13} \mathrm{C}(75.5 \mathrm{MHz}) \mathrm{NMR}$ measurements were recorded on a Bruker 300 spectrometer in DMSO- $\mathrm{d}_{6}$, acetone- $\mathrm{d}_{6}, \mathrm{CD}_{2} \mathrm{Cl}_{2}$ or $\mathrm{CDCl}_{3}$ using TMS as the internal reference. Mass spectra were recorded on a Varian Matt 311 spectrometer and relative abundances of fragments are quoted in parentheses after the $\mathrm{m} / \mathrm{z}$ values. Microanalyses were performed on a Leco Analyzer 932.

Bis diethyl (1,3-phenylene)thiocarbamoylmalonate (7a). To diethyl malonate $(1.58 \mathrm{~mL}, 10.44 \mathrm{mmol})$ in anhydrous THF $(20 \mathrm{~mL})$ was added sodium $(0.24 \mathrm{~g}, 10.44 \mathrm{mmol})$ and the mixture was refluxed under nitrogen for $2 \mathrm{~h}$. The solution was cooled to room temperature, 1,3 phenylenediisothiocyanate $(1 \mathrm{~g}, 5.22 \mathrm{mmol})$ was added dropwise, and the resulting yellow solution was stirred at room temperature for $3 \mathrm{~h}$. The mixture was extracted with $\mathrm{CH}_{2} \mathrm{Cl}_{2}(4 \times$ $20 \mathrm{~mL}$ ) and the aqueous phase was added dropwise to vigorously stirred, ice-cold $1 \mathrm{M} \mathrm{HCl}$ $(10 \mathrm{~mL})$, yielding a yellow oil. $(2.27 \mathrm{~g}, 85 \%) .{ }^{1} \mathrm{H}$ NMR $\left(\mathrm{CDCl}_{3}\right) \delta 1.33(\mathrm{t}, J=7.2 \mathrm{~Hz}, 12 \mathrm{H}), 4.31$ (q, $J=7.2 \mathrm{~Hz}, 8 \mathrm{H}), 5.07(\mathrm{~s}, 2 \mathrm{H}), 7.12(\mathrm{~d}, J=7.2 \mathrm{~Hz}, 1 \mathrm{H}), 7.38$ (t, $J=7.8 \mathrm{~Hz}, 1 \mathrm{H}), 7.6(\mathrm{~d}, J=7.8 \mathrm{~Hz}, 1 \mathrm{H})$, $7.72(\mathrm{t}, J=1.5 \mathrm{~Hz}, 1 \mathrm{H}), 10.85(\mathrm{~s}, 2 \mathrm{H}) ;{ }^{13} \mathrm{C} \mathrm{NMR}\left(\mathrm{CDCl}_{3}\right) \delta 13.91,63.16,67.35,117.7,120.11$, 122.95, 138.95, 165.62, 187.79; FT-IR (film) $v_{\max } / \mathrm{cm}^{-1}: 3269,2983,1747,1600,1556,1402$, 1306, 1025, 782; Anal. Calcd for $\mathrm{C}_{22} \mathrm{H}_{28} \mathrm{~N}_{2} \mathrm{~S}_{2} \mathrm{O}_{8}$ : C, 51.55; H, 5.51; N, 5.47\%; Found: C, 51.44; H, 5.12; N, 5.31 .

Bis diethyl (2-methyl-1,3-phenylene)thiocarbamoylmalonate (7b). This thiocarbamate was made by the same procedure. Diethyl malonate $(1.58 \mathrm{~mL}, 10.44 \mathrm{mmol})$ gave $7 \mathbf{b}(2.47 \mathrm{~g}, 90 \%)$ as yellow needles, mp 116-117 ${ }^{\circ} \mathrm{C} .{ }^{1} \mathrm{H}$ NMR $\left(\mathrm{CDCl}_{3}\right) \delta 1.35$ (t, J=7.2 Hz,12H), $2.83(\mathrm{~s}, 3 \mathrm{H}), 4.32$ (q, J=7.2 Hz, 8H), $5.15(\mathrm{~s}, 2 \mathrm{H}), 7.34(\mathrm{t}, J=7.9 \mathrm{~Hz}, 1 \mathrm{H}), 7.56(\mathrm{~d}, J=8.1 \mathrm{~Hz}, 2 \mathrm{H}), 10.55(\mathrm{~s}, 2 \mathrm{H}) ;{ }^{13} \mathrm{C}$ NMR $\left(\mathrm{CDCl}_{3}\right) \delta$ 12.43, 13.94, 63.14, 66.64, 125.99, 126.64, 130.97, 138.12, 165.62, 189.84; FT-IR (KBr) $v_{\max } / \mathrm{cm}^{-1}: 3271,2988,1754,1720,1519,1416,1309,1034,729$; Anal. Calcd for $\mathrm{C}_{23} \mathrm{H}_{30} \mathrm{~N}_{2} \mathrm{~S}_{2} \mathrm{O}_{8}$ : C, 52.46; H, 5.74; N, 5.32\%; Found: C, 51.21; H, 5.38; N, 5.31\%.

Bis ethyl 3-(1,3-phenylene)amino-5-oxo-2,5-dihydroisoxazole-4-carboxylate (8a). Hydroxyammonium chloride $(0.45 \mathrm{~g}, 7.8 \mathrm{mmol})$ was dissolved in ethanol $(8 \mathrm{~mL}) /$ water $(4 \mathrm{~mL})$ and then neutralized with potassium hydroxide $(0.54 \mathrm{~g}, 7.8 \mathrm{mmol}){ }^{8}$ The filtered solution was added to thiocarbamate $7 \mathrm{a}(1 \mathrm{~g}, 1.95 \mathrm{mmol})$ and the mixture was refluxed for $15 \mathrm{~h}$ before being cooled to room temperature. The reaction mixture was quenched with $1 \mathrm{M} \mathrm{HCl}(10 \mathrm{~mL})$, yielding a cream 
precipitate. The precipitate was recrystallised from ethanol to give $\mathbf{8 a}(0.56 \mathrm{~g}, 70 \%)$ as cream crystals, mp $214^{\circ} \mathrm{C}$ dec. ${ }^{1} \mathrm{H}$ NMR $\left(\mathrm{DMSO}_{6}\right) \delta 1.25(\mathrm{t}, J=7.2 \mathrm{~Hz}, 6 \mathrm{H}), 4.2(\mathrm{q}, J=7.2 \mathrm{~Hz}, 4 \mathrm{H})$, $7.14\left(\mathrm{dd}, J_{1}=8.1 \mathrm{~Hz}, J_{2}=4.5 \mathrm{~Hz}, 2 \mathrm{H}\right), 7.37(\mathrm{t}, J=7.8 \mathrm{~Hz}, 1 \mathrm{H}), 7.44(\mathrm{t}, J=1.8 \mathrm{~Hz}, 1 \mathrm{H}), 9.32(\mathrm{~s}, 2 \mathrm{H})$; ${ }^{13} \mathrm{C}$ NMR $\left(\mathrm{DMSO}_{\mathrm{d}}\right.$ ) $\delta$ 14.93, 59.46, 73.82, 114.07, 117.34, 130.62, 138.38, 162.62, 164.74, 167.19; FT-IR (KBr) $v_{\max } / \mathrm{cm}^{-1}: 3171,1778,1722,1672,1618,1582,1314,1183,1015,789$; $\mathrm{MS} \mathrm{m} / \mathrm{z}(\%) 418\left(\mathrm{M}^{+}, 15\right), 368(7), 210(16), 159(16), 132(30), 114(48), 83(84), 45(100)$.

Bis ethyl 3-(2-methyl-1,3-phenylene)amino-5-oxo-2,5-dihydroisoxazole-4-carboxylate (8b). This compound was prepared by the same procedure. Thiocarbamate $7 \mathbf{b}(1 \mathrm{~g}, 1.9 \mathrm{mmol})$ gave $\mathbf{8 b}$ $(0.66 \mathrm{~g}, 80 \%)$ as cream solid, $\mathrm{mp} 154-157^{\circ} \mathrm{C} .{ }^{1} \mathrm{H}$ NMR (Acetone-d $\left.{ }_{6}\right) \delta 1.31$ (t, $J=6.9 \mathrm{~Hz}, 6 \mathrm{H}$ ), 2.37 (s, 3H), 4.29 (q, J=6.9 Hz, 4H), 7.3 (t, J=8.1 Hz,1H), 7.47 (d, J=7.5 Hz, 2H), 9.35 (s, 2H);

${ }^{13} \mathrm{C}$ NMR (Acetone $-\mathrm{d}_{6}$ ) $\delta 11.89,13.90,59.43,74.41,123.45,125.57,135.86,164.97,165.07$, 165.47; FT-IR (KBr) $v_{\max } / \mathrm{cm}^{-1}: 3448,2988,1712,1673,1597,1433$, 795; Anal. Calcd for $\mathrm{C}_{19} \mathrm{H}_{20} \mathrm{~N}_{4} \mathrm{O}_{8}$ : C, 52.78; H, 4.66; N, 12.96\%; Found: C, 52.65; H, 4.22; N, 12.88\%.

Bis ethyl 3-(1,3-phenylene)amino-2-(pyrimidin-2-yl)-5-oxo-2,5-dihydroisoxazole-4carboxylate (9a). Isoxazolone 8a $(0.5 \mathrm{~g}, 1.19 \mathrm{mmol})$ and 2-chloropyrimidine $(0.272 \mathrm{~g}, 2.38 \mathrm{mmol})$ were heated neat under nitrogen at $130^{\circ} \mathrm{C}$ for $30 \mathrm{~min}$. The resulting product was recrystallised from ethanol to give the isoxaolone $9 \mathrm{a}(0.446 \mathrm{~g}, 65 \%)$ as brown crystals mp $162-164^{\circ} \mathrm{C} .{ }^{1} \mathrm{H}$ NMR $\left(\mathrm{DMSO}_{6}\right) \delta 1.15(\mathrm{t}, J=7.2 \mathrm{~Hz}, 6 \mathrm{H}), 4.1(\mathrm{q}, J=7.2 \mathrm{~Hz}, 4 \mathrm{H}), 6.85$ (d, J=7.2 Hz, 2H), 6.94 (t, $J=6.6 \mathrm{~Hz}, 1 \mathrm{H}), 7.21(\mathrm{~s}, 1 \mathrm{H}), 7.4(\mathrm{t}, J=5.1 \mathrm{~Hz}, 2 \mathrm{H}), 8.7(\mathrm{~d}, J=4.8 \mathrm{~Hz}, 4 \mathrm{H}), 10.33(\mathrm{~s}, 2 \mathrm{H}) ;{ }^{13} \mathrm{C}$ NMR $\left(\mathrm{DMSO}_{\mathrm{d}}\right.$ ) $\delta$ 14.62, 60.29, 78.99, 116.8, 119.21, 120.8, 129.4, 138.49, 156.59, 159.78, 161.36, 162.71, 164.02; FT-IR (KBr) $v_{\max } / \mathrm{cm}^{-1}: 3435,2925,1774,1696,1663,1594,1398,1177,1026$, 779; MS m/z (\%) 574( $\left.\mathrm{M}^{+}, 8\right)$, 530(31), 486(79), 440(37), 394(100), 365(86), 323(100), 277(100), 261(58), 205(62), 145(79), 89(100), 459(100).

Bis ethyl 3-(2-methyl-1,3-phenylene)amino-2-(pyrimidin-2-yl)-5-oxo-2,5-dihydroisoxazole4-carboxylate (9b). This compound was prepared by the same procedure. Isoxazolone $\mathbf{8 b}(0.5 \mathrm{~g}$, $1.16 \mathrm{mmol})$ gave 9b $(0.476 \mathrm{~g}, 70 \%)$ as brown crystals, mp 192-194 ${ }^{\circ} \mathrm{C} .{ }^{1} \mathrm{H} \mathrm{NMR}\left(\mathrm{CDCl}_{3}\right) \delta 1.35$ (t, $J=7.2 \mathrm{~Hz}, 6 \mathrm{H}), 2.48$ (s, 3H), 4.3 (q, J=7.2 Hz, 4H), 6.71 (t, $J=6.9 \mathrm{~Hz}, 1 \mathrm{H}), 6.9$ (d, $J=8.1 \mathrm{~Hz}$, 2H), $7.12(\mathrm{t}, J=4.8 \mathrm{~Hz}, 2 \mathrm{H}), 8.56(\mathrm{~d}, J=4.5 \mathrm{~Hz}, 4 \mathrm{H}), 10.13(\mathrm{~s}, 2 \mathrm{H}) ;{ }^{13} \mathrm{C}$ NMR $\left(\mathrm{CDCl}_{3}\right) \delta 13.10$, $14.40,60.85,78.95,119.09,120.40,125.78,126.14,137.36,155.94,158.52,161.23,163.31$, 164.45; FT-IR (KBr) $v_{\max } / \mathrm{cm}^{-1}: 3438,2977,1773,1710,1663,1600,1577,1397,1215,779$; Anal. Calcd for $\mathrm{C}_{27} \mathrm{H}_{24} \mathrm{~N}_{8} \mathrm{O}_{8}$ : C, 55.10; H, 4.11; N, 19.04\%; Found: C, 49.95; H, 4.22; N, $19.00 \%$.

Ethyl 2-[3-ethoxycarbonyl-2-(pyrimidin-2-ylamino)indol-6-yl]aminoimidazo[1,2a]pyrimidine-3-carboxlate (10a). The isoxazolone 9a $(0.1 \mathrm{~g}, 0.174 \mathrm{mmol})$ and triethylamine $(0.2 \mathrm{~mL})$ were refluxed in ethanol $(10 \mathrm{~mL})$ for $3 \mathrm{~h}$. On cooling, 10a crystallized as a cream solid $(0.072 \mathrm{~g}, 85 \%) \mathrm{mp} 205-207^{\circ}$ C. ${ }^{1} \mathrm{H}$ NMR $\left(\mathrm{DMSO}_{-} \mathrm{d}_{6}\right) \delta 1.17(\mathrm{t}, J=7 \mathrm{~Hz}, 3 \mathrm{H}), 1.41(\mathrm{t}, J=7 \mathrm{~Hz}, 3 \mathrm{H})$, 4.13 (q, $J=7 \mathrm{~Hz}, 2 \mathrm{H}), 4.43$ ( q, $J=7 \mathrm{~Hz}, 2 \mathrm{H}), 6.81(\mathrm{~d}, J=7.8 \mathrm{~Hz}, 1 \mathrm{H}), 7.13(\mathrm{t}, J=8.1 \mathrm{~Hz}, 1 \mathrm{H}), 7.27$ (t, $J=4.2 \mathrm{~Hz}, 1 \mathrm{H}), 7.37(\mathrm{t}, J=4.5 \mathrm{~Hz}, 1 \mathrm{H}), 7.52(\mathrm{~d}, J=7.8 \mathrm{~Hz}, 1 \mathrm{H}), 7.77(\mathrm{~s}, 1 \mathrm{H}), 8.61(\mathrm{bs}, 1 \mathrm{H})$, 8.72(d, J=4.5 Hz,3H), 9.29(bs,1H),10.39(bs,1H); ${ }^{13} \mathrm{C}$ NMR (DMSO-d 6 ) $\delta 14.64,14.89,60.19$, 60.82 , 78.32, 96.48, 110.88, 113.64, 116.12, 116.78, 120.88, 129.53, 136.04, 138.28, 140.95 , 
148.89, 152.47, 154.38, 157.07, 159.82, 160.77, 162.38, 162.96, 164.32; FT-IR (KBr) $v_{\max } / \mathrm{cm}^{-}$ 1: 3399, 2990, 1779, 1664, 1614, 1584, 1395, 1176, 1096, 761; MS m/z (\%) 486(M $\left.\mathrm{M}^{+}, 83\right)$, 485(100), 440(41), 392(63), 365(69), 205(55), 79(84), 78(100).

Ethyl 2-[3-ethoxycarbonyl-7-methyl-2-(pyrimidin-2-ylamino)indol-6-yl]aminoimidazo[1,2a]pyrimidine-3-carboxylate (10b). This compound was prepared by the same procedure. The isoxazolone 9b $(0.1 \mathrm{~g}, 0.17 \mathrm{mmol})$ gave $10 \mathrm{~b}(0.061 \mathrm{~g}, 72 \%)$ as cream solid, mp $230^{\circ} \mathrm{C} \mathrm{dec} .{ }^{1} \mathrm{H}$ $\operatorname{NMR}\left(\mathrm{CD}_{2} \mathrm{Cl}_{2}\right) \delta 1.32(\mathrm{t}, J=6.9 \mathrm{~Hz}, 3 \mathrm{H}), 1.53(\mathrm{t}, J=7.2 \mathrm{~Hz}, 3 \mathrm{H}), 2.44$ (s, 3H), 4.27 (q, J=6.9 Hz, 2H), 4.55 (q, J=7.2 Hz, 2H), 6.9 (d, J=7.7 Hz, 1H), 7.06 (d, J=4.5 Hz, 1H), 7.08 (t, J=4.5 Hz, $1 \mathrm{H}), 7.12$ (t, $J=4.8 \mathrm{~Hz}, 1 \mathrm{H}), 8.38$ (d, J=7.7 Hz, 1H), 8.57 (d, J=4.8 Hz, 3H), 9.31 (bs, 1H), 10.08 $(\mathrm{s}, 1 \mathrm{H}) ;{ }^{13} \mathrm{C}$ NMR $\left(\mathrm{CD}_{2} \mathrm{Cl}_{2}\right) \delta 12.33,14.09,14.53,60.54,60.82,78.05,96.65,109.6,112.37$, $117.73,117.93$, 119.30, 121.32, 126.65, 135, 136.4, 139.34, 151.32, 156.15, 158.45, 162.03; FTIR (KBr) $v_{\max } / \mathrm{cm}^{-1}: 3407,2982,1781,1686,1583,1480,1396,1103,1039,776$; Anal. Calcd for $\mathrm{C}_{25} \mathrm{H}_{24} \mathrm{~N}_{8} \mathrm{O}_{4}$ : C, 59.99; H, 4.83; N, 22.39\%; Found: C, 59.54; H, 4.39; N, 22.23\%.

\section{Acknowledgements}

We are grateful to professor R.H.Prager (Flinders University) for his valuable comments and gift of chemicals. We also thank Urmia University for financial support.

\section{References}

1. Jeffery, D.; Prager, R. H.; Turner, D.; Dreimanis, M. Tetrahedron 2002, 58, 9965.

2. Khalafy, J.; Molla Ebrahimlo, A. R.; Eisavi, R.; Akbari Dilmaghani, K. Arkivoc 2005, xiv, 59.

3. Khalafy, J.; Poursattar Marjani, A.; Molla Ebrahimlo, A. R. J. Braz. Chem. Soc. 2006, 17, 570

4. Khalafy, J.; Molla Ebrahimlo, A. R.; Akbari Dilmaghani, K. J. Chin. Chem. Soc. 2004, 51, 1347.

5. Khalafy, J.; Prager, R. H.; Smith, J. A. J. Chem. Res(M). 1999,518.

6. Pham, T.-N; Tuteja, R.; Ocham, A.; Falaschi, A. Biochem. Biophys. Res. Commun. 1997, 236, 636.

7. Stiborova, M.; Bieler, C. A.; Wiessler, M.; Frei, E. Biochem. Pharmacol. 2001, 62, 1675.

8. Worrall, D. E. J. Am. Chem. Soc. 1923, 45, 3092.

9. Khalafy, J.; Prager, R. H. J. Sci. I. R. Iran 2000, 11, 32.

10. Perrin, D. D.; Armarego, W. L. F. Purification of Laboratory Chemicals, Pergamon Press: Oxford, U.K., 1988. 